\title{
The Real-life Experience of Developing and Commercializing TruGraf, a Validated Non-invasive Transplant Biomarker
}

\author{
M Roy First ${ }^{1,2 *}$, Steven B Kleiboeker ${ }^{3}$, Stanley Rose ${ }^{1}$, John J Friedewald ${ }^{2}$, and Michael M Abecassis ${ }^{4}$ \\ ${ }^{1}$ Transplant Genomics Inc, USA \\ ${ }^{2}$ Northwestern University, Comprehensive Transplant Center, USA \\ ${ }^{3}$ Viracor Eurofins Clinical Diagnostics, USA \\ ${ }^{4}$ University of Arizona College of Medicine-Tucson, USA
}

*Corresponding author: M Roy First, Transplant Genomics Inc, Northwestern University, Comprehensive Transplant Center, USA, Tel: 7736778682; E-mail: roy@transplantgenomics.com

Received: 24 Apr, 2020 | Accepted: 11 May, 2020 | Published: 18 May, 2020

Citation: First MR, Kleiboeker SB, Rose S, Friedewald JJ, Abecassis MM (2020) The Real-life Experience of Developing and Commercializing TruGraf, a Validated Non-invasive Transplant Biomarker. J Biochem Analyt Stud 4(2): dx.doi.org/10.16966/2576-5833.123

Copyright: (C) 2020 First MR, et al. This is an open-access article distributed under the terms of the Creative Commons Attribution License, which permits unrestricted use, distribution, and reproduction in any medium, provided the original author and source are credited.

\begin{abstract}
Despite improvement in short-term outcomes, long-term results for kidney transplant recipients remain suboptimal. Immunological rejection is a leading cause of graft failure and recent research points to undetected "silent" subclinical acute rejection as a key component of this problem. While biopsies remain the gold-standard method for detecting silent rejection, non-invasive methods offer significant advantages especially in terms of patient safety and for serial monitoring of stable patients. This manuscript details the real-life challenges involved in the ultimately successful development and commercialization of TruGraf, a clinically validated, blood-based gene expression assay that offers the potential to reduce the use of surveillance (protocol) biopsies in renal transplant recipients with stable renal function.
\end{abstract}

Keywords: Non-invasive transplant biomarker; TruGraf; Kidney transplantation; Biopsies; Renal function

\section{Introduction}

Kidney transplantation is the optimal treatment for most patients with chronic renal failure [1]. However, the long term success of kidney transplantation is far from optimal [2]. In 2017, 10-year all-cause graft failure was $49.7 \%$ for deceased donor kidney recipients and $34.1 \%$ for living donor kidney transplants [3]. Immunological rejection, a major cause of graft failure, is driven by attack of the graft by $\mathrm{T}$ cells ( $\mathrm{T}$ cell mediated rejection, or TCMR) or antibodies (antibody mediated rejection, or ABMR), or in some cases a combination of these two mechanisms (mixed rejection). A key early contributor to long-term graft loss is subclinical immune injury that leads to chronic damage of the renal allograft [4-8]. Until recently there have been no commercially available fully validated non-invasive tests to monitor patients with stable renal function for silent rejection [9]. As a result, a significant number of centers rely on surveillance (protocol) biopsies to detect early silent rejection, whereas other centers who choose not to perform these wait for clinical evidence of graft injury and damage $[10,11]$.

\section{Situational Analysis}

Standard non-invasive monitoring to detect kidney injury secondary to rejection or other causes includes measuring serum creatinine levels and immunosuppressive drug levels, both of which are insensitive and nonspecific. Clinical manifestations of severe rejection, such as fever, pain over the graft, or decreased urine output may be present, but are infrequent findings with current immunosuppressive regimens. Thus, current non-invasive monitoring only detects rejection when it is advanced and only after significant, and potentially irreversible damage to the graft has occurred. Indication or for cause biopsies are typically performed to determine the cause of acute renal dysfunction.

Biopsies are expensive, invasive, and suffer from significant variability in interpretation [12]. Moreover, biopsies put patients at risk for significant complications such as infection, bleeding, and even graft loss, in addition to being painful and inconvenient [13]. However, indication biopsies remain essential in the management of patients with renal dysfunction and are used ubiquitously by transplant programs. In sharp contrast, while a number of transplant programs have adopted the routine use of surveillance biopsies to detect subclinical acute rejection (subAR) in patients with stable renal function, several factors have discouraged other programs from following suit. These include but are not limited to all the issues stated above, but, in addition, stable patients undergo indiscriminate biopsies resulting in negative (unnecessary) invasive procedures the vast majority of the time. Thus, a noninvasive monitoring strategy that replaces invasive protocol biopsies is sorely needed and has been the focus of several investigators in the past few years. 
Previous investigators focused on developing non-invasive biomarkers in the urine and blood to diagnose rejection in patients with graft dysfunction (clinical acute rejection-cAR) in an attempt to replace indication biopsies. There are two major fallacies to this approach: first, while some patients with subAR develop cAR, others exhibit ongoing subAR causing more chronic graft injury; second, in the absence of paired biopsies for each sample, it is difficult to be certain that bio-informatics approaches which yield positive results from these samples are real. For this reason, we set out to develop a biomarker specific for subAR by using only blood samples paired with protocol biopsies in patients with stable renal function.

\section{Development of a validated peripheral blood biomarker for subAR}

Identifying the need for a non-invasive replacement for biopsies in stable patients, we set out to discover and validate a peripheral blood biomarker to detect subAR in these patients as a "rule in" test, similar to biopsies. While our clinical trials and sample collection regimens were well designed, the evidentiary data and biomarker performance that resulted caused us to rethink the context of use (COU) of the biomarker.

Subclinical acute rejection (subAR), also referred to as "silent" rejection, is histologically defined acute rejection characterized by tubulointerstitial mononuclear cell infiltration identified from a biopsy specimen in a patient with normal or stable renal function [4-8]. In the NIH-sponsored CTOT-08 study of 307 kidney transplant recipients [7], the natural prevalence of subAR, based on surveillance biopsies, was $20 \%$ at 3-6 months, and $25 \%$ at 12 and 24 months surveillance biopsies, with an overall prevalence of $35 \%$ [7]. Of note, $80 \%$ of the subAR was of the borderline variety when classified by central pathology using the Banff criteria [14], and importantly, the biopsy was normal in $75 \%$ of cases. At the two year time point, patients with subAR on surveillance biopsies had worse outcomes than patients who did not. This was based on a composite clinical endpoint (CCE) consisting of biopsy-proven acute rejection (BPAR) on any "forcause biopsy" by central read, or a 24-month biopsy (central read) showing evidence of chronic injury measured by interstitial fibrosis and tubular atrophy (IFTA) of Banff grade $\geq$ II IFTA (ci $\geq 2$ or ct $\geq 2$ ), or a decrease in estimated glomerular filtration rate (GFR) by $>10 \mathrm{~mL} /$ $\mathrm{min} / 1.73 \mathrm{~m}^{2}$ between 4 and 24 months post-transplant [7]. SubAR was also associated with a higher frequency of both class I and class II de novo donor specific antibody (dnDSA) development $[7,15]$.

In addition to the CTOT-08 data shown above, a number of clinical studies have also recently associated subAR with poor outcomes [4$8,15-19]$. A study in recipients with a rapid steroid withdrawal protocol compared outcomes in patients with no inflammation and those with subclinical inflammation on a 3-month surveillance biopsy. In the patients with subclinical inflammation, the serum creatininelevels were significantly higher at 24 months, and the allograft chronicity index on biopsy, the rate of subsequent BPAR and development of dnDSA were all significantly increased at 12 months [16]. A large Australian study compared outcomes in patients with normal biopsies, those with borderline rejection, and those with $\mathrm{T}$ cell mediated acute rejection. Compared to patients with normal biopsies, patients with borderline rejection had worse renal function, more IFTA, subsequent acute rejection, allograft failure and patient mortality [7]. A recent study in 103 pediatric renal transplant recipients that examined subclinical inflammation phenotypes and long-term outcomes after pediatric kidney transplantation, highlights the importance and treatment of subAR [18]. In this study, surveillance biopsies were performed in first 6 months and a composite endpoint (CEP) of acute rejection and graft failure was measured at 5 years. The CEP was reached by $41 \%$ for treated borderline rejection $v s .67 \%$ for untreated $(p<0.001)$ [18]. Additionally, another recent publication has shown that borderline early acute rejection is associated with the development of late acute rejection and graft loss [19].

\section{The Trials and Tribulations of a) developing and b) commercializing a non-invasive biomarker for subAR}

Development: The TruGraf Blood Gene Expression Test (Transplant Genomics, Inc, Mansfield, MA) is a microarray-based assay that analyzes gene expression profiles (GEP) in the peripheral blood. Our initial strategy was to develop a "rule in" test, whereby a positive test would be highly predictive of a positive biopsy (subAR). We used a locked support vector machine (SVM) based classifier with a bootstrap to prevent over-fitting of the discovery set for internal validation as the bio-informatics approach [20]. We found two interesting observations: first, at different thresholds, we traded PPV for NPV to the point that a "rule in" test was not possible using this approach. We then switched to Random Forest (RF) as the bioinformatics approach [21] and used a different threshold, but again it was evident that the intended use of the biomarker would need to change. Because the performance metrics were better with RF, we proceeded to use RF but picked thresholds more favourable for a "rule out" test [21]. The product was a GEP classifier that associates with either a normal protocol kidney biopsy (Transplant eXcellence-TX) or the absence of a normal biopsy (not-TX) in patients with stable renal function. All aspects of discovery and external validation of the TruGraf test were performed on blood samples paired with biopsies from prevalent cohorts. For the purpose of validation, the model derived from pre-selected bio-informatics and the threshold used to test performance on the discovery cohort were locked. These data led us to use this approach for external validation in an early access program (EAP) for patients [22]. The external clinical validation from seven EAP transplant centers defined the key clinical performance parameters for this assay, as summarized in table 1 and figure 1 . In this study, the high negative predictive value (NPV) of TruGraf was demonstrated in clinical use, making it a strong rule-out test. Over $90 \%$ of stable patients who received a TX results were confirmed to have an immune quiescent phenotype, meaning that a physician can have a high degree of confidence that a patient who tests as TX does not harbour silent subclinical rejection. Importantly this study also found that up to $65 \%$ of surveillance biopsies could be avoided in the cohort tested. Unpublished data involving analysis of an additional 129 biopsy-confirmed blood samples provided by Northwestern University (originally used for the CTOT-08 study) revealed identical performance metrics for TruGraf (NPV of 90\%). A fourth publication described the impact of TruGraf results on physician decision making for clinical decisions [23]. This study highlighted the high degree of confidence physicians place in the ability of TruGraf to provide valuable, added information that could lead to avoidance of unnecessary surveillance biopsies as summarized in table 2 . In the prospective study $(n=45) 87.0 \%$ of physician responses indicated that the result of the TruGraf test supported their management decision in a patient with stable renal function, with a corresponding rate in the retrospective study $(\mathrm{n}=192)$ of $87.5 \%$ [23].

As a result of these experiences, we changed the proposed COU from replacing surveillance biopsies for detecting subAR, to reducing the number of necessary biopsies in stable patients which should lead to many less invasive procedures (Table 1 ) as well as significantly less negative or unnecessary biopsies. The COU proposed in the recent approval from CMS states that "The TruGraf test is intended for use in 
Table 1: Summary of TruGraf v1 performance characteristics in a multicenter, observational study [22].

\begin{tabular}{|c|c|c|c|c|c|c|c|c|}
\hline & \multirow[b]{2}{*}{$\mathbf{N}$} & \multirow[b]{2}{*}{ Sensitivity } & \multirow[b]{2}{*}{ Specificity } & \multirow[b]{2}{*}{ PPV } & \multirow[b]{2}{*}{ NPV } & \multicolumn{2}{|c|}{$\begin{array}{c}\text { Adjusted to } 24.5 \% \text { subAR } \\
\text { prevalence }\end{array}$} & \multirow{2}{*}{$\begin{array}{c}\text { \% TX (biops } \\
\text { spared) }\end{array}$} \\
\hline & & & & & & PPV & NPV & \\
\hline All samples & 192 & $76.5 \%$ & $73.4 \%$ & $38 \%$ & $93.5 \%$ & $48 \%$ & $91 \%$ & $65 \%$ \\
\hline $\begin{array}{l}\text { Paired blood and biopsy } \\
\text { subset }\end{array}$ & 99 & $70.8 \%$ & $74.7 \%$ & $47 \%$ & $89 \%$ & $48 \%$ & $89 \%$ & $64 \%$ \\
\hline
\end{tabular}

NPV=negative predictive value;

$\mathrm{PPV}=$ positive predictive value.

Table 2: Responses from principal investigators to prospective and retrospective questionnaires [22].

\begin{tabular}{|c|c|c|c|}
\hline Timing of Questionnaire & Clinical Utility Feedback & Yes & No \\
\hline \multirow{2}{*}{ Prospective $(n=45)$} & $\begin{array}{c}\text { Did the TruGraf result support your decision on how to manage a patient with stable serum } \\
\text { creatinine? }\end{array}$ & $39(87 \%)$ & $6(13 \%)$ \\
\hline & $\begin{array}{c}\text { Does the TruGraf result encourage you to use TruGraf serial testing in future patient } \\
\text { management? }\end{array}$ & $42(93 \%)$ & $3(7 \%)$ \\
\hline Retrospective ( $n=192)$ & $\begin{array}{c}\text { Would this result have had any impact on your management of your patient in terms of } \\
\text { maintaining or changing immunosuppression, changing frequency of clinic visits, or deciding } \\
\text { on Whether to do a biopsy or not? }\end{array}$ & $168(87.5 \%)$ & $24(12.5 \%)$ \\
\hline
\end{tabular}

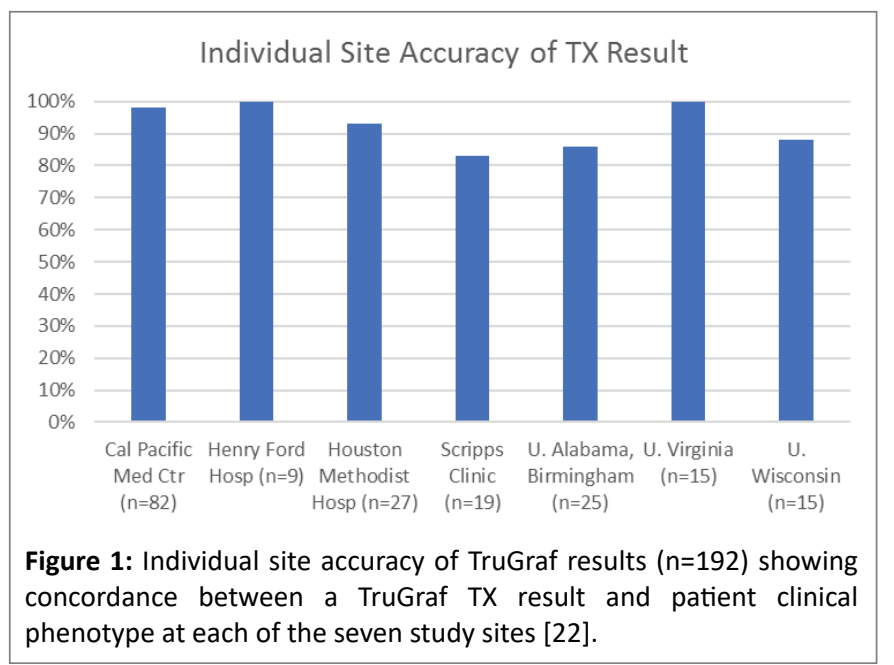

kidney transplant recipients with stable renal function as an alternative to surveillance biopsies in facilities that utilize surveillance biopsies". While primarily used to rule out subAR, it is expected that both centers that perform or do not perform surveillance biopsies can use the test to assess the need for a surveillance biopsy in stable patients [24].

Figure 2 illustrates a proposed approach for implementation of TruGraf into clinical care for kidney transplant recipients. For patients with stable renal function, a TruGraf result of "TX" identifies those who have a high likelihood of immune quiescence and a low likelihood of histologically defined rejection at the borderline level or higher. A result of "Not-TX" identifies those in whom silent rejection cannot be confidently ruled out, and thus carry a higher risk of immune activation and borderline or higher rejection. Patients with a "Not-TX" result might benefit from further evaluation and possibly a change in therapy. Early identification of these patients potentially allows better allocation of physician resources, and potential reversal of the process before permanent damage to the donated kidney occurs.

Pathway to commercialization of TruGraf: Developed in 2011, the Molecular Diagnostic Services (MolDX) program is run by Palmetto

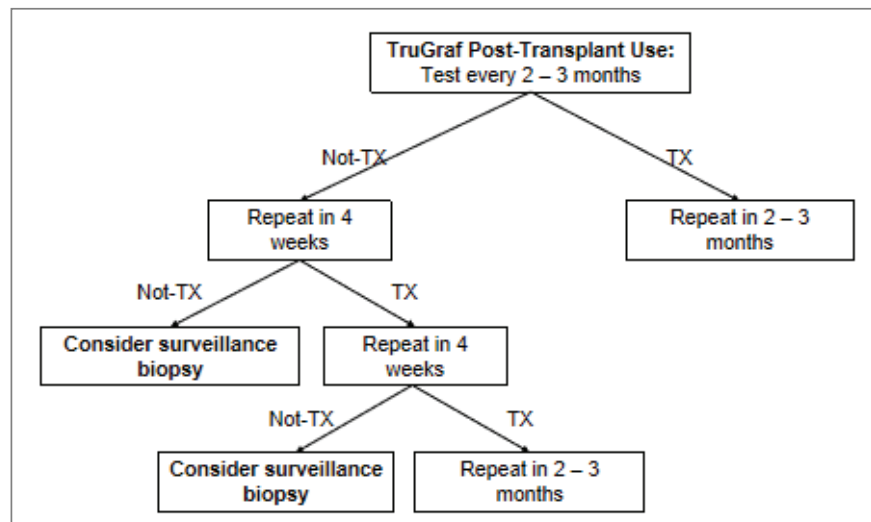

Figure 2: Suggested use of TruGraf for kidney transplant recipients in the first 5 years post-transplant. Frequency of testing and clinical use of results is based on expert opinion. TX=Transplant excellence, indicating immunological quiescence in the transplanted kidney; NotTx=abnormal result.

GBA, a centers for Medicare and Medicaid Services (CMS) Medicare administrative contractor. It performs the following functions:

- Facilitates detailed and unique identification through registration of molecular diagnostics tests to facilitate claims processing and to track utilization.

- Establishes clinical utility expectations. Completes technical assessments of published test data to determine clinical utility and coverage.

CMS approved reimbursement for commercial TruGraf testing on November 25, 2019.

A recently published study evaluated the clinical validity of serial TruGraf testing in stable kidney transplant recipients in a center not utilizing surveillance biopsies [25]. Serum creatinine levels and TruGraf testing at multiple time points were correlated with clinical follow-up in 28 patients. The concordance of TruGraf results, when compared with independent clinical assessment of testing, was $77 \%$ 
(54/70) for all tests; $79 \%(22 / 28)$ for test $1,75 \%(21 / 28)$ for test 2 , and $79 \%(11 / 14)$ for test 3 . The NPV in this study was $98.0 \%$, and analysis of clinical utility indicated that $77 \%$ of TruGraf results would have aided in patient management. These results indicate the value of serial TruGraf testing in those transplant centers that do not perform surveillance biopsies as part of their standard of care. The high NPV confirms immune quiescence with a high degree of probability in patients with a TruGraf test result of TX without the need to perform a biopsy.

\section{Conclusions}

Silent subclinical rejection is frequent and a significant contributor to worse long term outcomes for kidney transplant recipients. Until now subAR could only be ruled in or out by invasive and risky per protocol surveillance biopsies, resulting in a significant number of unnecessary biopsies and therefore unnecessary risk to patients compromising safety. Thus, non-invasive tests are clearly needed to identify patients with stable renal function who are harbouring subAR in their grafts. In response to this statement of need, we first set out to develop a "rule in" test to replace the routine use of protocol biopsies as the context of use. However, based on the evidentiary performance data of our biomarker, we determined that it is best used as a "rule out" test and then revised the proposed COU as the reduction of a large proportion of protocol biopsies in programs that currently utilize these; in those that do not, subjecting far fewer patients to the risks of biopsies together with a reduction in the number of unnecessary (negative) biopsies may provide an attractive monitoring strategy [24]. To these ends, TruGraf is the first and only non-invasive test designed and validated for use in ruling out silent subclinical rejection in kidney transplant recipients with stable renal function.

Non-invasive blood testing can be done more frequently than surveillance kidney biopsies, is significantly less invasive, less painful and risky for patients, and may result in a considerable cost savings to the health delivery system.

\section{Conflict of Interest}

MRF, SBK and SR are full-time employees of Eurofins-Transplant Genomics, who developed the TruGraf test described in this manuscript. JJF and MMA are consultants to Eurofins-Transplant Genomics.

\section{References}

1. Rana A, Gruessner A, Agopian VG, Khalpey Z, Riaz IB, et al. (2015) Survival benefit of solid-organ transplant in the United States. JAMA Surg 150: 252-259.

2. Lamb KE, Lodhi S, Meier-Kriesche HU (2011) Long-term renal allograft survival in the United States: a critical reappraisal. Am J Transplant 11: 450-462.

3. Hart A, Smith JM, Skeans MA, Gustafson SK, Wilk AR, et al. (2019) OPTN/SRTR 2017 Annual Data Report: Kidney. Am J Transplant 19: 19-123.

4. Nankivell BJ, Chapman JR (2006) The significance of subclinical rejection and the value of protocol biopsies. Am J Transplant 6: 2006-2012.

5. Kuypers DR (2008) Immunosuppressive drug therapy and subclinical acute renal allograft rejection: impact and effect. Transplantation 85: S25-S30.

6. Loupy A, Vernerey D, Tinel C, Aubert O, Duong van Huyen JP, et al. (2015) Subclinical rejection phenotypes at 1 year post-transplant and outcome of kidney allografts. Am J Soc Nephrol 26: 1721-1731.
7. Friedewald JJ, Kurian SM, Heilman RL, Whisenant TC, Poggio ED, et al. (2019) Development and clinical validity of a novel blood-based molecular biomarker for subclinical acute rejection following kidney transplant. Am J Transplant 19: 98-109.

8. Hoffman W, Mehta R, Jorgensen DR, Sood P, Randhawa P, et al. (2019) The Impact of Early Clinical and Subclinical T Cell-mediated Rejection After Kidney Transplantation. Transplantation 103: 14571467.

9. Waldmann H (2014) Drug minimization in transplantation: an opinion. Curr Opin Organ Transplant 19: 331-333.

10. Metha R, Cherikh W, Sood P, Hariharan S (2017) Kidney allograft surveillance biopsy practices across US transplant centers: A UNOS survey. Clin Transplant 31.

11. Lee DM, Abecassis MM, Friedewald JJ, Rose S, First MR, et al. (2019) Kidney Graft Surveillance Biopsy Utilization and Trends-Results from a Survey of High Volume Transplant Centers. Abstract: American Transplant Congress, Boston, MA.

12. Mengel M, Sis B, Halloran PF (2007) SWOT analysis of Banff: strengths, weaknesses, opportunities and threats of the international Banff consensus process and classification system for renal allograft pathology. Am J Transplant 7: 2221-2226.

13. Morgan TA, Chandran S, Burger IM, Zhang CA, Goldstein RB (2016) Complications of Ultrasound-Guided Renal Transplant Biopsies. Am J Transplant 16: 1298-1305.

14. Haas M, Loupy A, Lefaucheur C, Roufosse C, Glotz D, et al. (2018) The Banff 2017 Kidney Meeting Report: Revised diagnostic criteria for chronic active T cell-mediated rejection, antibody- mediated rejection, and prospects for integrative endpoints for nextgeneration clinical trials. Am J Transplant 18: 293-307.

15. R Heilman, D Taber, K Guo, S Brietigam, j charette, et al. (2019) Subclinical Rejection by Biopsy or Gene Expression Profile Predicts Long Term Kidney Function in Patients in the СтОт08 Trial. Am J Transplant 19.

16. Mehta R, Bhusal S, Randhawa P, Sood P, Cherukuri A, et al. (2018) Short-term adverse effects of early subclinical allograft inflammation in kidney transplant recipients with a rapid steroid withdrawal protocol. Am J Transplant 18: 1710-1717.

17. Nankivell BJ, Agrawal N, Sharma A, Taverniti A, $\mathrm{P}^{\prime} \mathrm{Ng} \mathrm{CH}$, et al. (2018) The clinical and pathological significance of borderline T cell mediated rejection. Am J Transplant 19: 1452-1463.

18. Seifert ME, Yanik MV, Feig DI, Hauptfeld-Dolejsek V, MroczekMusulman EC, et al. (2018) Subclinical inflammation phenotypes and long-term outcomes after pediatric kidney transplantation. Am J Transplant 18: 2189-2199.

19. Zhang W, Yi Z, Wei C, Keung KL, Sun Z, et al. (2019) Pretransplant transcriptomic signature in peripheral blood predicts early acute rejection. JCl Insight 4: 127543.

20. Martin Roy First, Deirdre Pierry, Michael McNulty, Sunil M Kurian, Stan Rose, et al. (2017) Analytical and clinical validation of a molecular diagnostic signature in kidney transplant recipients. J Transplant Technol Res 7: 1-9.

21. First MR, Whisenant T, Friedewald JJ, Lewis P, Rose S, et al. (2017) Clinical utility of peripheral blood gene expression profiling of kidney transplant recipients to assess the need for surveillance biopsies in subjects with stable renal function. J Transplant Technol Res 7: 1-6. 
22. Marsh CL, Kurian SM, Rice JC, Whisenant TC, David J, et al. (2019) Application of TruGraf v1: A Novel Molecular Biomarker for Managing Kidney Transplant Recipients With Stable Renal Function. Transplant Proc 51: 722-728.

23. First MR, Peddi VR, Mannon R, Knight R, Marsh CL, et al. (2019) Investigator assessment of the utility of the TruGraf molecular diagnostic test in clinical practice. Transplant Proc 51: 729-733.
24. Friedewald J, Abecassis M (2019) Clinical implications for the use of a biomarker for subclinical rejection - Conflating arguments cause a disconnection between the premise and the conclusion. Am J Transplant 19: 2141-2142.

25. Peddi VR, Patel PS, Schieve C, Rose S, First MR (2020) Serial Peripheral Blood Gene Expression Profiling in Kidney Transplant Recipients with Stable Renal Function to Assess Immune Quiescence. Ann Transplant 25: e920839. 